\title{
Damage-Based Approach for bimodular and anisotropic materials
}

\author{
Formulación basada en daño para materiales teniendo en cuenta la Bimodularidad y \\ Anisotropía
}

José Julio de Cerqueira Pituba ${ }^{1}$

Recibido 15 de marzo de 2017, aceptado 19 de febrero de 2018

Received: March 15, 2017 Accepted: February 19, 2018

\begin{abstract}
This work intends to present the development of a formulation based on Continuum Damage Mechanics to deal with elastic media introducing different mechanical behaviors in tension and compression regimes as well as some anisotropy degree when damage processes take place. First of all, a formulation for bimodular and anisotropic elastic media is reviewed. To take into account the damage processes in the material, some improvements are proposed as well as the definition of a hyperplane in order to divide the strain space to deal with bimodular materials. Besides, fourth-order anisotropic tensors are requested by the formulation depending on the class of anisotropy. Soon after, two models of constitutive damage written accordingly with the formulation proposed in this work are described. Finally, it is shown that the proposed formulation is an easy way to write and to understand complex constitutive damage models.
\end{abstract}

Keywords: Damage mechanics, anisotropy, constitutive model.

\section{RESUMEN}

Este trabajo pretende presentar el desarrollo de una formulación basada en Mecánica de Daños para tratar con medios elásticos que presentan comportamientos mecánicos diferentes en regímenes de tensión y compresión, así como algún grado de anisotropía cuando se producen procesos de daño. En primer lugar, se revisa una formulación para medios elásticos bimodulares y anisotrópicos. Con el fin de tener en cuenta los procesos de daño en el material, se proponen algunas mejoras así como la definición de un hiperplano para dividir el espacio de deformación para tratar con materiales bimodulares. Además, los tensores anisotrópicos de cuarto orden son solicitados por la formulación dependiendo de la clase de anisotropía. Poco después, se describen dos modelos constitutivos de daños escritos en concordancia con la formulación propuesta en este trabajo. Finalmente, se muestra que la formulación propuesta es una forma fácil de escribir y comprender los complejos modelos de daño.

Palabras clave: Mecánica de daño, anisotropía, modelo constitutivo.

1 Laboratory of Computational Modeling. Engineering School. Federal University of Goias. Av. Dr. Lamartine Pinto de Avelar 1120.75740-220. Catalão, Goiás, Brasil. E-mail: julio_pituba@ufg.br 


\section{INTRODUCTION}

Many fiber-reinforced composite materials behave like anisotropic and bimodular media. On the other hand, brittle materials belong to a class of materials that can be considered initially isotropic and unimodular, such as concrete. However, these materials start to present different behaviors in tension and compression when damaged. In this context, considering small deformations, a formulation of constitutive laws for isotropic and anisotropic elastic materials presenting different behaviors in tension and compression regimes has been proposed in [1] to two and threedimensional cases.

The formulation presented in this work intends to introduce some improvements in the work [1] To take into account the damage effects in the mechanical behavior of materials. It is shown that the formulation is able to reproduce some damage models proposed in the literature, as for instance, [2-3].

Many different strategies are possible and they have been proposed in the literature to model bimodular materials and stiffness recovery as described in [4-5], [3], [6-10] and [11]. For more details, in is presented a summary of some model formulations which take into account the unilateral effect of the damage process.

On the other hand, the Continuum Damage Mechanics (CDM) has already proved to be a suitable tool for simulating the material deterioration in equivalent continuous media due exclusively to microcracking process. In the CDM, the damage effects are evidenced in the stiffness constitutive tensor. The damage process leads to the reduction of several stiffness components, where the damaged material can either keep its isotropic properties or become anisotropic, [2]. In this context, some isotropic damage models applied to the analysis of concrete have been developed, as [3], [12-13]. However, in the last decades anisotropic models which can modify both, the direction and the number of the material symmetry planes, have been proposed using phenomenological or micromechanical approaches ([2], [14-20] and others). For example, in [10], a micromechanical approach has been proposed to explain the anisotropic behavior of the macrostructure as a result of specific microcrack patterns. Moreover, many works dealing with the prediction of mechanical behavior of materials using information about two or more physical scales [21-26].

Despite the approach is based on phenomenological features or microstructure information of the material, the proposed formulation is presented as an alternative to be used as a proper tool to formulate damage models and to easily write easily these kind of models.

Finally, this work is divided into six sections. Initially, a linear elasticity formulation for bimodular and anisotropic media is briefly presented. After that, the extended framework incorporating the damage effects is discussed and some considerations about the representation of constitutive tensors for isotropic and anisotropic materials are presented. As a result, two damage models are written based on the formalism presented in this work. In particular, a bi-dissipative anisotropic damage model proposed for the non-linear analysis of reinforced concrete structures is derived from the formulation presented in this work. A criterion for partition of strain space is proposed. Finally, a few conclusions are discussed in the last section.

\section{THEORETICAL ANALYSES}

\section{Linear elasticity for anisotropic and bimodular media}

In this section, a linear elasticity formulation for anisotropic media considering different behaviors in tension and compression regimes is briefly presented. For more details, see [1].

Considering the hyperelasticity, a linear stress-strain law deriving from a quadratic potential involving a constant elasticity tensor presents the following characteristics:

$$
\begin{array}{ll}
W(\varepsilon)=\frac{1}{2} \varepsilon: E \varepsilon & W \in \ell^{2}(\varepsilon, \Re) \\
\sigma(\varepsilon)=E \varepsilon & \sigma \in \ell^{1}(\varepsilon, \wp) \\
\boldsymbol{E}=\text { constant } & \boldsymbol{E} \in \ell^{0}(\varepsilon, \boldsymbol{E})
\end{array}
$$

In equation (1), $W$ is an elastic potential energy density which must be twice continuously differentiable. In equation (2), $\sigma$ is a second-order tensor once differentiable and $E$ is a fourth-order tensor. In the 
general case of anisotropy, the constitutive tensor $E$ is entirely determined by 21 independent constants.

The previous relationships for the anisotropic linear elasticity involve the coefficients named bulk $\left(\lambda_{a b}\right)$ and shear $\left(\mu_{a}\right)$ moduli which are reduced to those named Lamè constants in the isotropy cases. The particular forms that include isotropy, transverse isotropy and orthotropy, are given by:

$$
\begin{gathered}
W(\varepsilon)=\frac{\lambda_{a b}}{2} \operatorname{tr}\left(A_{a} \varepsilon\right) \operatorname{tr}\left(A_{b} \varepsilon\right)+ \\
\mu_{a} \operatorname{tr}\left(A_{a} \varepsilon^{2}\right) \quad(a, b=1, d) \\
\sigma(\varepsilon)=E \varepsilon \quad \sigma \in \ell^{1}(\varepsilon, \wp) \\
E=\lambda_{a b}\left[A_{a} \otimes A_{b}\right]+\mu_{a}\left[A_{a} \otimes I+I \otimes-\right. \\
\left.\lambda_{a b}=\lambda_{b a}\right]
\end{gathered}
$$

where $d=1$ for isotropy (recovering then the two usual Lamé constants), $d=2$ for transverse isotropy ( 5 constants) and $d=3$ for orthotropy ( 9 constants). The tensors $A_{a}$ and $A_{b}$ are defined as: $\left(A_{l}=I\right)$ for isotropic materials, $\left(A_{1}=I, A_{2}=A\right)$ for transverse isotropic materials and $\left(A_{1}=I, A_{2}=A, A_{3}=B\right)$ for orthotropic materials.

For example, in the transverse isotropy cases, the tensor $I$ is the second-order identity tensor and the tensor $A$, by definition, Curnier et al. (1995), is formed by dyadic product of the unit vector perpendicular to the transverse isotropy plane for himself. More details about the products are given in [27] and in the appendix.

However, for the one-dimensional case, the word "bimodulus" is related to the materials whose onedimensional stress-strain law is characterized by different Young's moduli in tension and compression regimes.

In [1] is proposed an extension of that characteristic to two and three-dimensional cases. In the sequel, the development of that work is described.

For the modeling of a bimodular hyperelastic material, the elastic potential energy density $\mathrm{W}$ must be once continuously differentiable (whole wise), but only piecewise twice continuously differentiable. The stress-strain relationship derived from this potential is piecewise continuously differentiable leading to an elasticity tensor discontinuous referred to a hypersurface that contains the origin and divides the strain space into a compression and tension sub-domains. In this way, the modeling is able to reproduce different responses in tension and compression regimes.

Therefore, let the strain space $\varepsilon$ be divided into compression sub-domain $\varepsilon$. and tension sub-domain $\varepsilon_{+}$by means of a hypersurface $I$ characterized by a scalar valued function $g(\varepsilon)$ (Figure 1). Hence, there are defined the following representations:

$$
\begin{aligned}
\mathbb{\&}:=\left\{\varepsilon \in \varepsilon \mid g(\varepsilon)=0 g \in \ell^{1}(\varepsilon, \Re)\right. \\
\mathcal{\varepsilon}_{-}:=\{\varepsilon \in \varepsilon \mid g(\varepsilon)<0\}, \\
\overline{\boldsymbol{\varepsilon}}_{-}:=\{\varepsilon \in \varepsilon \mid g(\varepsilon) \leq 0\} \\
\varepsilon_{+}:=\{\varepsilon \in \varepsilon \mid g(\varepsilon)>0\}, \\
\overline{\boldsymbol{\varepsilon}}_{+}:=\{\varepsilon \in \varepsilon \mid g(\varepsilon) \geq 0\} \\
\mathcal{\varepsilon}=\boldsymbol{\varepsilon}_{-} \cup \mathcal{E}_{+} \cup \boldsymbol{\&}, \\
\boldsymbol{\varepsilon}_{-} \cap \varepsilon_{+}=\varnothing, \quad \overline{\boldsymbol{\varepsilon}}_{-} \cap \overline{\boldsymbol{\varepsilon}}_{+}=\boldsymbol{\&}
\end{aligned}
$$

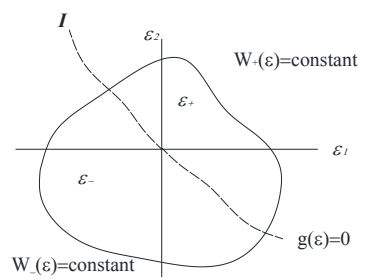

Figure 1. Partition of strain space into compression and tension sub-domains.

The hypersurface must contain the strain space origin in order to:

$$
0 \in \& \Leftrightarrow g(0)=0
$$

The definition of the hypersurface is used as criterion in order to divide the strain space into compression and tension dominant regimes. For instance, the simplest subdivision of the strain space into two half-spaces is provided by a hyperplane characterized by its normal unit (second-order tensor) $\mathrm{N}(\|\mathrm{N}\|=1)$. Therefore, the expressions below are valid: 


$$
\begin{gathered}
\&:=\{\varepsilon \in \varepsilon \mid g(\varepsilon)=N . \varepsilon=0\} \\
\varepsilon_{-}:=\{\varepsilon \in \varepsilon \mid N . \varepsilon<0\}, \\
\varepsilon_{+}:=\{\varepsilon \in \varepsilon \mid N . \varepsilon>0\}
\end{gathered}
$$

The material response can be separately described in compression and tension dominant regimes by two elastic energy functions $\mathrm{W}_{-}(\varepsilon)$ and $\mathrm{W}_{+}(\varepsilon)$. These functions are restricted to their relevant sub-domains $\varepsilon_{\text {. and }} \varepsilon_{+}$, besides when juxtaposed they provide a single energy function $W(\varepsilon)$ defined over $\varepsilon$.

More specifically, the following relationships are valid:

$$
\begin{aligned}
& W(\varepsilon):=\left\{\begin{array}{l}
W_{-}(\varepsilon) \text { if } g(\varepsilon) \leq 0, W_{-} \in \ell^{2}(\varepsilon, \mathfrak{R}), \\
W_{+}(\varepsilon) \text { if } g(\varepsilon) \geq 0, W_{+} \in \ell^{2}(\varepsilon, \mathfrak{R}),
\end{array}\right. \\
& W \in \ell^{1}(\varepsilon, \mathfrak{R}) \\
& \sigma(\varepsilon)=\nabla_{\varepsilon} W(\varepsilon)= \\
& \left\{\begin{array}{l}
\sigma_{-}(\varepsilon)=\nabla_{\varepsilon} W_{-}(\varepsilon) f \\
\sigma_{+}(\varepsilon)=\nabla_{\varepsilon} W_{+}(\varepsilon) f \\
g(\varepsilon) \leq 0 \\
g(\varepsilon) \geq 0
\end{array}\right.
\end{aligned}
$$$$
\boldsymbol{E}(\varepsilon)= \begin{cases}E_{-}(\varepsilon)=\nabla_{\varepsilon}^{2} W_{-}(\varepsilon) \text { if } & g(\varepsilon)<0 \\ E_{+}(\varepsilon)=\nabla_{\varepsilon}^{2} W_{+}(\varepsilon) \text { if } & g(\varepsilon)>0\end{cases}
$$

where

$$
\begin{aligned}
& W(0)=W_{-}(0)=W_{+}(0)=0 \\
& \sigma(0)=\sigma_{-}(0)=\sigma_{+}(0)=0
\end{aligned}
$$

The continuity of elastic energy function and its first gradient (stress-strain law) across the interface implies

$$
\begin{aligned}
& W(\varepsilon)=W_{+}(\varepsilon)=W_{-}(\varepsilon) \\
& \forall \varepsilon \mid g(\varepsilon)=0 \\
& \sigma(\varepsilon)=\sigma_{+}(\varepsilon)=\sigma_{-}(\varepsilon) \\
& \forall \varepsilon \mid g(\varepsilon)=0
\end{aligned}
$$

However, the elasticity tensor presents a jump across the interface in the normal direction defined by $\nabla g$, but there is no tangential discontinuity. The jump in the elasticity tensor can be given by:

$$
\begin{aligned}
& {[E(\varepsilon)]:=E_{+}(\varepsilon)-E_{-}(\varepsilon)=} \\
& s(\varepsilon) \nabla g(\varepsilon) \otimes \nabla g(\varepsilon) \\
& \forall \varepsilon \mid g(\varepsilon)=0
\end{aligned}
$$

where $s(\varepsilon)$ is a continuous scalar valued function that provides the jump value.

Using the hyperplane, the invariant form of $g(\varepsilon)$ must be a linear combination of the linear invariants $I_{a}=\operatorname{tr}\left(A_{a}\right.$ $\varepsilon)$, i.e., $g(\varepsilon)=\gamma_{a} I_{a}$, and its constant gradient $\nabla \varepsilon$ $g(\varepsilon)=N$, the normal vector to the hyperplane must be a linear combination of the corresponding tensors $A_{\boldsymbol{a}}$. In general cases, the following relationships are valid:

$$
\begin{gathered}
\varepsilon]=\begin{array}{l}
g(\varepsilon)=\gamma_{a} \operatorname{tr}\left(A_{a} \varepsilon\right)=\operatorname{tr}\left[\left(\gamma_{a} A_{a}\right)\right. \\
N . \varepsilon=0, \quad(a=1, d), \\
N=\nabla_{\varepsilon} g=\frac{\partial g}{\partial \mathrm{I}_{a}} \nabla_{\varepsilon} \mathrm{I}_{a}=\gamma_{a} A_{a},
\end{array}
\end{gathered}
$$

where coefficients $\gamma_{a}$ implies: $\| N||=1$

Finally, the expressions for the energy function equation (14), for the stress-strain law equation (15) and elasticity tensor equation (16) are written in the following forms:

$$
\begin{aligned}
& W(g(\varepsilon), \varepsilon)=\frac{\lambda_{a b}(g(\varepsilon))}{2} \operatorname{tr}\left(A_{a} \varepsilon\right) \\
& \operatorname{tr}\left(A_{b} \varepsilon\right)+\mu_{a} \operatorname{tr}\left(A_{a} \varepsilon^{2}\right) \\
& (a, b=1, d) \\
& \sigma(g(\varepsilon), \varepsilon)=\lambda_{a b}(g(\varepsilon)) \operatorname{tr}\left(A_{a} \varepsilon\right) A_{b} \\
& +\mu_{a}\left(A_{a} \varepsilon+\varepsilon A_{a}^{T}\right) \\
& E(g(\varepsilon), \varepsilon)=\lambda_{a b}(g(\varepsilon))\left[A_{a} \otimes A_{b}\right. \\
& +\mu_{a}\left[A_{a} \otimes-\bar{\otimes}_{-} I_{-} A_{a}\right]
\end{aligned}
$$


The bimodular character implies that the constants $\lambda_{a b}$ are dependent of $g(\varepsilon)$, i.e.:

$$
\lambda_{a b}(g(\varepsilon))=\lambda_{b a}:=\left(\begin{array}{lll}
\lambda_{a b}^{-} & \text {if } & g(\varepsilon)<0 \\
\lambda_{a b}^{+} & \text {if } & g(\varepsilon)>0
\end{array}\right.
$$

It must be pointed out that the coefficients $\mu_{a}$ are the same in tension and compression regimes.

\section{Extended formulation for anisotropic elastic media with damage and bimodular response}

In this section, the extended framework of [1] incorporating the damage effect is presented. Accordingly, the coefficients named bulk $\left(\lambda_{a b}\right)$ and shear $\left(\mu_{a}\right)$ moduli are considered as functions of the damage state, where the stress-strain relationship would be influenced by damage variables. Moreover, the hypersurface $g(\varepsilon, D i)$ taken as a criterion for the identification of the constitutive responses in compression or tension regimes would also be influenced by the damage variables. Note that the condition about no tangential discontinuity of the elasticity tensor is also valid in this formulation.

A more specific aspect to be highlighted out is related to the thermodynamically associated variables to the damage ones. Such variables are energy released rates throughout the damage evolution process and they can be used in the definition of a criterion to identify the beginning and evolution of the damage process.

On the other hand, the choice of the damage variables as well as the anisotropy class considered in a constitutive model formulation depends on the material to be modelled. In this context, it is important to observe that the damage variables influence the change of the fourth-ordertensors $\left[\mathrm{A}_{\mathrm{a}} \otimes \mathrm{A}_{\mathrm{b}}\right]$ and $\left[\mathrm{A}_{\mathrm{a}} \bar{\otimes} \mathrm{I}+\mathrm{I} \otimes \mathrm{A}_{\mathrm{a}}\right]$ which are included in the elasticity tensor expressions.

Then, the relations for the extended potential energy function and derived relationships valid for general cases of anisotropy can be written in the following form:

$$
\begin{aligned}
& W\left(D_{i}, g(\varepsilon), \varepsilon\right)=\frac{\lambda_{a b}\left(D_{i}, g(\varepsilon)\right)}{2} \\
& \operatorname{tr}\left(\boldsymbol{A}_{a} \boldsymbol{\varepsilon}\right) \operatorname{tr}\left(\boldsymbol{A}_{b} \boldsymbol{\varepsilon}\right)+\mu_{a}\left(D_{i}\right) \operatorname{tr}\left(\boldsymbol{A}_{a} \boldsymbol{\varepsilon}^{2}\right)
\end{aligned}
$$

$$
\begin{aligned}
& \boldsymbol{E}\left(D_{i}, g(\varepsilon), \varepsilon\right)=\lambda_{a b}\left(D_{i}, g(\varepsilon)\right) \\
& {\left[A_{a} \otimes A_{b}\right]+\mu_{a}\left(D_{i}\right)\left[A_{a} \bar{\otimes} I+I \bar{\otimes} A_{a}\right]} \\
& \boldsymbol{E}\left(D_{i}, g(\varepsilon), \varepsilon\right)=\lambda_{a b}\left(D_{i}, g(\varepsilon)\right) \\
& {\left[A_{a} \otimes A_{b}\right]+\mu_{a}\left(D_{i}\right)\left[A_{a} \otimes I+I \otimes-\overline{-} A_{a}\right]}
\end{aligned}
$$

where the subscript $i$ can assume values from 1 to the number of damage scalar variables considered by the model.

The variables associated to the damage ones can be obtained from the energy function, as follows:

$$
\begin{aligned}
& \mathrm{Y}\left(\mathrm{Di}, \mathrm{g}\left(\varepsilon, \mathrm{D}_{\mathrm{i}}\right), \varepsilon\right)=\nabla_{D} W=\frac{\partial W}{\partial D_{i}}= \\
& -\frac{1}{2} \frac{\partial \lambda_{a b}\left(D_{i}, g\left(\varepsilon, D_{i}\right)\right)}{\partial D_{i}} \\
& \operatorname{tr}\left(A_{a} \cdot \varepsilon\right) \operatorname{tr}\left(A_{b} \cdot \varepsilon\right) \\
& \quad-\frac{\partial \mu_{a}\left(D_{i}\right)}{\partial D_{i}} \operatorname{tr}\left(A_{a} \cdot \varepsilon^{2}\right)
\end{aligned}
$$

Note that the bimodular character implies on the $\lambda_{a b}$ coefficients are functions of the $g(\varepsilon, D i)$, i.e.:

$$
\begin{aligned}
& \lambda_{a b}\left(D_{i}, g\left(\varepsilon, D_{i}\right)\right)=\lambda_{b a}:=\left(\begin{array}{l}
\lambda_{a b}^{-}\left(D_{i}\right) \\
\lambda_{a b}^{+}\left(D_{i}\right)
\end{array}\right. \\
& \text { if } g\left(\varepsilon, D_{i}\right)<0 \\
& \text { if } g\left(\varepsilon, D_{i}\right)>0
\end{aligned}
$$

It must be pointed out that the conditions above impose a jump in the elasticity tensor across the hypersurface, $g(\varepsilon, D i)$. Besides, the shear coefficients $\mu_{a}$ must be the same in tension and compression regimes, as explained in [1].

Actually, the choice for the number of damage variables and class of anisotropy depends on the material that will be modeled. In this context, this formulation can be extended to include a more complex level of anisotropy. Therefore, the proposed formulation can be employed for damaged elastic materials where the medium is initially anisotropic and bimodular. Also, the proposed formulation can be used for damaged elastic materials with anisotropy and bimodularity induced by damage. In the last case, it must be careful with the insert of 
the damage variables in the formulation to induce anisotropy and bimodularity conveniently. That possibility is explored in the next section, to show the potentialities of the proposed formulation.

\section{Formulation for damaged elastic media taking into account induced transverse isotropy and bimodular response}

Consider now that the material initially behaves as an isotropic medium with the same stiffness in tension and compression regimes. When the damage process takes place, the material starts to present a bimodular and anisotropic behavior. Then, by assuming a generic situation that the medium already presents a certain damage level leading to a transverse isotropy behaviour, the elastic energy potential and the derived relationships to stress, constitutive tensors, and variables associated to damage are given by:

$$
\begin{aligned}
& \mathrm{W}\left(\mathrm{D}_{\mathrm{i}}, \mathrm{g}\left(\varepsilon, D_{i}\right), \varepsilon\right)=\frac{\lambda_{11}}{2} \operatorname{tr}^{2}(\varepsilon) \\
& +\mu_{1} \operatorname{tr}\left(\varepsilon^{2}\right)-\frac{\lambda_{22}\left(D_{i}, g\left(\varepsilon, D_{i}\right)\right)}{2} \operatorname{tr}^{2}(\mathrm{~A} \varepsilon) \\
& \text { - } \lambda_{12}\left(D_{i}, g\left(\varepsilon, D_{i}\right)\right) \operatorname{tr}(\varepsilon) \operatorname{tr}(A \varepsilon) \\
& -\mu_{2}\left(D_{i}\right) \operatorname{tr}\left(A \varepsilon^{2}\right) \\
& \sigma\left(\mathrm{D}_{\mathrm{i}}, \mathrm{g}\left(\varepsilon, \mathrm{D}_{\mathrm{i}}\right), \varepsilon\right)=\lambda_{11} \operatorname{tr}(\varepsilon) \mathrm{I} \\
& +2 \mu_{1} \varepsilon-\lambda_{22}\left(D_{i}, g\left(\varepsilon, D_{i}\right)\right) \operatorname{tr}(\mathrm{A} \varepsilon) \mathrm{A} \\
& \text { - } \lambda_{12}\left(D_{i}, g\left(\varepsilon, D_{i}\right)\right)[\operatorname{tr}(\varepsilon) \mathrm{A} \\
& +\operatorname{tr}(\mathrm{A} \varepsilon) \mathrm{I}]-\mu_{2}\left(\mathrm{D}_{\mathrm{i}}\right)\left[\mathrm{A} \varepsilon+\varepsilon \mathrm{A}^{\mathrm{T}}\right] \\
& \mathrm{E}\left(\mathrm{D}_{\mathrm{i}}, \mathrm{g}\left(\varepsilon, \mathrm{D}_{\mathrm{i}}\right), \varepsilon\right)=\mathrm{E}_{0} \\
& \text { - } \lambda_{22}\left(D_{i}, g\left(\varepsilon, D_{i}\right)\right)[A \otimes A] \\
& -\lambda_{12}\left(\mathrm{D}_{\mathrm{i}}, \mathrm{g}\left(\varepsilon, \mathrm{D}_{\mathrm{i}}\right)\right)[\mathrm{A} \otimes \mathrm{I}+\mathrm{I} \otimes \mathrm{A}] \\
& -\mu_{2}\left(\mathrm{D}_{\mathrm{i}}\right)[\mathrm{A} \otimes \overline{\mathrm{I}}+\mathrm{I} \otimes \mathrm{A}] \\
& \mathrm{Y}\left(\mathrm{D}_{\mathrm{i}}, \mathrm{g}\left(\varepsilon, \mathrm{D}_{\mathrm{i}}\right), \varepsilon\right)=\nabla W=\frac{\partial W}{\partial D_{i}}= \\
& -\frac{1}{2} \frac{\partial \lambda_{22}\left(D_{i}, g\left(\varepsilon, D_{i}\right)\right)}{\partial D_{i}} t r^{2}(A \varepsilon) \\
& -\frac{\partial \lambda_{12}\left(D_{i}, g\left(\varepsilon, D_{i}\right)\right)}{\partial D_{i}} \operatorname{tr}(\varepsilon) \operatorname{tr}(\mathrm{A} \varepsilon) \\
& -\frac{\partial \mu_{2}\left(D_{i}\right)}{\partial D_{i}} \operatorname{tr}\left(\mathrm{A} \varepsilon^{2}\right)
\end{aligned}
$$

where $\lambda_{11}=\lambda_{0}$ and $\mu_{1}=\mu_{0}$ are Lamè constants and $E_{0}$ is the initial isotropic elastic stiffness tensor.

The bimodular character is taken into account by the following conditions:

$$
\begin{aligned}
& \lambda_{12}\left(D_{i}, g\left(\varepsilon, D_{i}\right)\right):=\left(\begin{array}{l}
\lambda_{12}^{-}\left(D_{i}\right) \text { if } \\
\lambda_{12}^{+}\left(D_{i}\right) \text { if }
\end{array}\right. \\
& g\left(\varepsilon, D_{i}\right)<0 \\
& g\left(\varepsilon, D_{i}\right)>0 \\
& \lambda_{22}\left(D_{i}, g\left(\varepsilon, D_{i}\right)\right):=\left(\begin{array}{l}
\lambda_{22}^{-}\left(D_{i}\right) \text { if } \\
\lambda_{22}^{+}\left(D_{i}\right) \text { if }
\end{array}\right. \\
& g\left(\varepsilon, D_{i}\right)<0 \\
& g\left(\varepsilon, D_{i}\right)>0
\end{aligned}
$$

Note also that for null values of the damage variables the material behaves as an isotropic and unimodular medium.

\section{Representation of constitutive tensors for isotropic and anisotropic materials}

In the Continuum Damage Mechanics, the damage effects are evidenced in the constitutive tensor. The damage process leads to a reduction of several stiffness components, where the damaged material can keep its isotropic properties or become anisotropic. In the isotropic constitutive models, damage does not affect the number of both, the symmetry directions and the initial symmetry planes of the material, i.e., if the medium is initially isotropic or contains some anisotropy level, those characteristics are preserved throughout the damage process.

On the other hand, the anisotropic models have the capability to change the number of both: the symmetry directions and the initial symmetry planes of the material. In the last years, many constitutive models have been proposed in order to take into account the anisotropic characteristic of the medium, [7] and [28].

In order to represent the constitutive formulation for media with anisotropy induced by damage, it is considered that the damaged stiffness constitutive tensor can be composed by two additive parts: the first one represents the undamaged material and, the second one, is dependent of the actual damage state. When that last part is written like a summation of the scalar valued functions of the damage scalar variables that multiplies the fourth-order constitutive 
tensors, the resulting model is so-called scalar damage model. For instance, simple additive forms can be expressed as follows:

$$
\boldsymbol{E}=\boldsymbol{E}_{\boldsymbol{0}}+\lambda[D] \bar{M}
$$

where $\boldsymbol{E}_{0}$ and $\bar{M}$ are fourth-order tensors and $\lambda[D]$ is a scalar valued function of the damage scalar variables. The equation above is a condensed form of the eq. (35). For initially isotropic materials, it can preserve the isotopic characteristic or create an anisotropic effect on the constitutive tensors depending how the tensors $\boldsymbol{E}_{\boldsymbol{0}}$ and $\bar{M}$ have been defined.

On the other hand, the general forms for the fourthorder damage tensor $\boldsymbol{D}$ can be proposed in order to take into account the anisotropy induced by damage. In this work the definition of that tensor follows a so-called scalar form expressed as: $\boldsymbol{D}=f_{j}\left(D_{i}\right) \boldsymbol{M}_{\boldsymbol{j}}$, where $f_{j}\left(D_{i}\right)$ are scalar valued functions of the damage scalar variables $D_{i}$ and $\boldsymbol{M}_{\boldsymbol{j}}$ are anisotropic tensors.

\section{RESULTS}

This section intends to present some constitutive damage models found in the literature following the extended framework of [1] described in this work.

\section{A damage model accounting for induced anisotropy and bimodular elastic response}

The material is assumed as an initially isotropic material that starts to present transverse isotropy and bimodular responses induced by the damage. To take into account the bimodularity conveniently, two damage tensors governing the stiffness in tension or compression regimes are introduced. Moreover, the model tries to respect the principle of energy equivalence between damaged real medium and equivalent continuous medium established in the Continuum Damage Mechanics, [29]. Thus, the rigidity and flexibility constitutive tensors of the equivalent continuous medium result symmetric. Later, a criterion for partition of strain space is also discussed.

Following the proposed formulation and the principles of the irreversible processes Thermodynamics [29], the invariant form of the constitutive model is given by:

$$
\begin{gathered}
W(\varepsilon)=\rho \psi(\varepsilon):=\left\{\begin{array}{l}
W_{-}(\varepsilon) \\
W_{+}(\varepsilon)
\end{array}\right. \\
\text { if } g\left(\varepsilon, D_{T}, D_{C}\right)<0, \\
\text { if } g\left(\varepsilon, D_{T}, D_{C}\right)>0, \\
\text { and } W_{-}=W_{+}=W_{0} \text { if } \boldsymbol{D}_{T}=\boldsymbol{D}_{C}=\mathbf{0} \\
\mathrm{W}_{+}=\rho \psi_{+}(\varepsilon)=\frac{\lambda_{11}}{2} \operatorname{tr}^{2}(\varepsilon)+\mu_{1} \operatorname{tr}\left(\varepsilon^{2}\right) \\
-\frac{\lambda_{22}^{+}\left(D_{1}, D_{4}, D_{5}\right)}{2} \operatorname{tr}^{2}(A \varepsilon) \\
\quad-\lambda_{12}^{+}\left(D_{1}\right) \operatorname{tr}(\varepsilon) \operatorname{tr}(A \varepsilon) \\
\quad-\mu_{2}\left(D_{4}, D_{5}\right) \operatorname{tr}\left(A \varepsilon^{2}\right) \\
\mathrm{W}_{-}=\rho \psi_{-}(\varepsilon)=\frac{\lambda_{11}}{2} \operatorname{tr}^{2}(\varepsilon)+\mu_{1} \operatorname{tr}\left(\varepsilon^{2},\right. \\
-\frac{\lambda_{22}^{-}\left(D_{2}, D_{3}, D_{4}, D_{5}\right)}{2} \operatorname{tr}^{2}(A \varepsilon) \\
-\frac{\lambda_{12}^{-}\left(D_{2}, D_{3}\right) \operatorname{tr}(\varepsilon) \operatorname{tr}(A \varepsilon)}{-\frac{\lambda_{11}^{-}\left(D_{3}\right)}{2} \operatorname{tr}^{2}(\varepsilon)-\frac{\left(1-2 v_{0}\right)}{2 v_{0}} \lambda_{11}^{-}\left(D_{3}\right)} \\
\operatorname{tr}\left[\left(I \otimes \frac{\otimes}{\otimes} I\right) \varepsilon\right]^{2}-\mu_{2}\left(D_{4}, D_{5}\right) \operatorname{tr}\left(A \varepsilon^{2}\right)
\end{gathered}
$$

where $W_{0}$ is the elastic potential of the undamaged material, $\psi$ is the free energy potential, $\varrho$ is the mass density of the media, $\lambda_{\mathbb{1}}=\lambda_{0}$ and $\mu_{1}=\mu_{0}$ . The remaining parameters $\lambda$ and $\mu$ will only exist for non-zero damage, evidencing in that way the anisotropy and bimodularity induced by damage. Those parameters are given by:

$$
\begin{aligned}
& \lambda_{22}^{+}\left(D_{1}, D_{4}, D_{5}\right)=\left(\lambda_{0}+2 \mu_{0}\right) \\
& \left(2 D_{1}-D_{1}^{2}\right) \\
& -2 \lambda_{12}^{+}\left(D_{1}\right)-2 \mu_{2}\left(D_{4}, D_{5}\right) \\
& \lambda_{12}^{+}\left(D_{1}\right)=\lambda_{0} D_{1} \\
& \mu_{2}\left(D_{4}, D_{5}\right)=2 \mu_{0}\left[1-\left(1-D_{4}\right)^{2}\right. \\
& \left.\left(1-D_{5}\right)^{2}\right] \\
& \lambda_{22}^{-}\left(D_{2}, D_{3}, D_{4}, D_{5}\right)=\left(\lambda_{0}+2 \mu_{0}\right) \\
& \left(2 D_{2}-D_{2}^{2}\right)-2 \lambda_{12}^{-}\left(D_{2}, D_{3}\right) \\
& +\frac{\left(v_{0}-1\right)}{v_{0}} \lambda_{11}^{-}\left(D_{3}\right)-2 \mu_{2}\left(D_{4}, D_{5}\right) \\
& \lambda_{12}^{-}\left(D_{2}, D_{3}\right)=\lambda_{0}\left[\left(1-D_{3}\right)^{2}\right. \\
& \left.\quad-\left(1-D_{2}\right)\left(1-D_{3}\right)\right] \lambda_{11}^{-}\left(D_{3}\right)= \\
& \quad \lambda_{0}\left(2 D_{3}-D_{3}^{2}\right)
\end{aligned}
$$


The stress tensor is obtained from the gradient of the elastic potential, as follows:

$$
\begin{gathered}
\sigma(\varepsilon)=\nabla_{\varepsilon} \rho \psi(\varepsilon)= \\
\left\{\begin{array}{l}
\sigma_{-}(\varepsilon)=\nabla_{\varepsilon} \rho \psi_{-}(\varepsilon) \text { if } \\
\sigma_{+}(\varepsilon)=\nabla_{\varepsilon} \rho \psi_{+}(\varepsilon) \text { if }
\end{array}\right. \\
g\left(\varepsilon, D_{T}, D_{C}\right)<0, \\
g\left(\varepsilon, D_{T}, D_{C}\right)>0, \\
\sigma_{+}(\varepsilon)=\rho \frac{\partial \psi_{+}}{\partial \varepsilon}=\lambda_{11} \operatorname{tr}(\varepsilon) \mathrm{I}+2 \mu_{1} \varepsilon \\
-\lambda_{22}^{+}\left(\mathrm{D}_{1}, \mathrm{D}_{4}, \mathrm{D}_{5}\right) \operatorname{tr}(\mathrm{A} \varepsilon) \mathrm{A} \\
-\lambda_{12}^{+}\left(D_{1}\right)(\operatorname{tr}(\varepsilon) A+\operatorname{tr}(A \varepsilon) I) \\
-\mu_{2}\left(D_{4}, D_{5}\right)(A \varepsilon+\varepsilon A) \\
\sigma_{-}(\varepsilon)=\rho \frac{\partial \psi_{-}}{\partial \varepsilon}=\lambda_{11} \operatorname{tr}(\varepsilon) \mathrm{I} \\
+2 \mu_{1} \varepsilon-\lambda_{22}^{-}\left(\mathrm{D}_{2}, \mathrm{D}_{3}, \mathrm{D}_{4}, \mathrm{D}_{5}\right) \operatorname{tr}(\mathrm{A} \varepsilon) \mathrm{A} \\
-\lambda_{12}^{-}\left(D_{2}, D_{3}\right)(\operatorname{tr}(\varepsilon) A+\operatorname{tr}(A \varepsilon) I) \\
-\lambda_{11}^{-}\left(D_{3}\right) \operatorname{tr}(\varepsilon) I-\frac{\left(1-2 v_{0}\right)}{v_{0}} \lambda_{11}^{-}\left(D_{3}\right) \\
(I \otimes I) \varepsilon-\mu_{2}\left(D_{4}, D_{5}\right)(A \varepsilon+\varepsilon A)
\end{gathered}
$$

The constitutive tensor is also obtained from the elastic potential, i.e.:

$$
\begin{gathered}
\boldsymbol{E}(\varepsilon):=\left\{\begin{array}{l}
E_{-}(\varepsilon)=\nabla_{\varepsilon}^{2} \rho \psi_{-}(\varepsilon) \text { if } \\
E_{+}(\varepsilon)=\nabla_{\varepsilon}^{2} \rho \psi_{+}(\varepsilon) \text { if }
\end{array}\right. \\
g\left(\varepsilon, D_{T}, D_{C}\right)<0, \\
g\left(\varepsilon, D_{T}, D_{C}\right)>0, \\
E_{+}(\varepsilon)=\rho \frac{\partial^{2} \psi \psi_{+}}{\partial \varepsilon^{2}}=E_{T}= \\
\lambda_{11}[I \otimes I]+2 \mu_{1}[I \bar{\otimes} I] \\
-\lambda_{22}^{+}\left(D_{1}, D_{4}, D_{5}\right)[A \otimes A] \\
-\lambda_{12}^{+}\left(D_{1}\right)[A \otimes I+I \otimes A] \\
-\mu_{2}\left(D_{4}, D_{5}\right)[A \underline{\otimes} I+I \otimes \bar{\otimes} A]
\end{gathered}
$$

The variables associated to damage variables in tension $\left(D_{1}, D_{4}\right)$ and in compression $\left(D_{2}, D_{3}, D_{5}\right)$ regimes can be understood like energy release rates during the damage evolution process. They are given by:

$$
\begin{aligned}
& Y_{1}\left(D_{1}\right)=-\rho \frac{\partial \psi_{+}}{\partial D_{1}} ; Y_{2}\left(D_{1}\right)=\rho \frac{\partial \psi_{-}}{\partial D_{2}} ; \\
& Y_{3}\left(D_{3}\right)=-\rho \frac{\partial \psi_{-}}{\partial D_{3}} ; \\
& Y_{4}\left(D_{4}\right)=-\rho \frac{\partial \psi_{+}}{\partial D_{4}} ; Y_{5}\left(D_{5}\right)=-\rho \frac{\partial \psi_{-}}{\partial D_{5}}
\end{aligned}
$$

The constitutive model includes two damage tensors to take into account the bimodularity induced by damage in the concrete behavior. Therefore, a criterion is necessary to define the tension and compression dominant states in order to indicate what damage tensor should be used.

In this model, a particular form is adopted for the hypersurface in the strain space: a hyperplane $g(\varepsilon)$ defined by the unit normal $N(\|N\|=1)$ and characterized by its dependence on the strain and damage states.

This criterion is extended so that the actual damage state can influence the hyperplane definition. Therefore, the following relationship is proposed:

$$
g\left(\varepsilon, D_{T}, D_{C}\right)=N\left(D_{T}, D_{C}\right) . \varepsilon^{e}
$$

The strain space division in this model is discussed as follows. In this context, it is interesting to remember 
the Curnier equations equation (22) and equiation (23) related to the strain space division. For the isotropy case, the sub-index " $a$ " must be equal to $l$ and the tensor $A_{l}=I$. On this condition with damage absence, the null volumetric elastic strain defines the interface between the two strain sub-domains. In fact,

$$
\begin{gathered}
N=\gamma_{1} A_{1}=\gamma_{1} I, \quad(\mid \boldsymbol{N} \|=1 \\
\left.\Leftrightarrow \gamma_{1}=\frac{1}{\sqrt{3}}\right) \\
g(\varepsilon)=N \cdot \varepsilon^{e}=0, \\
g(\varepsilon)=\frac{1}{\sqrt{3}} \boldsymbol{I} . \varepsilon^{e}=\frac{1}{\sqrt{3}} \operatorname{tr}\left(\varepsilon^{e}\right)= \\
\frac{1}{\sqrt{3}} \varepsilon_{V}^{e}=0, \text { thus } \varepsilon_{V}^{e}=0
\end{gathered}
$$

where $\varepsilon_{\mathrm{V}}^{\mathrm{e}}$ is the volumetric elastic strain.

Furthermore, for the transverse isotropy case without damage, in equation (22) the sub-index and the tensors $\boldsymbol{A}_{\boldsymbol{a}}$ are: $a=1,2, \boldsymbol{A}_{\boldsymbol{I}}=\boldsymbol{I}$ and $\boldsymbol{A}_{2}=\boldsymbol{A}$. This implies:

$$
\begin{gathered}
N=\gamma_{1} A_{1}+\gamma_{2} A_{2}=\gamma_{1} \cdot I+\gamma_{2} \cdot A \\
g(\varepsilon)=N \cdot \varepsilon=0 \\
g(\varepsilon)=\left(\gamma_{1} I+\gamma_{2} A\right) \cdot \varepsilon^{e}= \\
\gamma_{1}\left(I \cdot \varepsilon^{e}\right)+\gamma_{2} A \cdot \varepsilon^{e}= \\
\gamma_{1} \operatorname{tr}\left(\varepsilon^{e}\right)+\gamma_{2} \operatorname{tr}\left(A \cdot \varepsilon^{e}\right)=0 \\
\mathrm{~g}(\varepsilon)=\gamma_{1} \varepsilon_{\mathrm{V}}^{\mathrm{e}}+\gamma_{2} \\
\left(\varepsilon_{11}^{\mathrm{e}} \mathrm{a}_{1}^{2}+\varepsilon_{22}^{\mathrm{e}} \mathrm{a}_{2}^{2}+\varepsilon_{33}^{\mathrm{e}} \mathrm{a}_{3}^{2}\right)=0
\end{gathered}
$$

where $a_{1}, a_{2}$ and $a_{3}$ are components of the unit vector normal to the transverse isotropy plane, $\gamma_{1}$ and $\gamma_{2}$ implies that $\|N\|=1$.

To simplify the presentation, the hyperplane will be here expressed as the one obtained by enforcing the direction $l$ in the strain space to be perpendicular to the transverse isotropy local plane. Thus, the components $a_{2}$ and $a_{3}$ result null and $a_{1}=1$ and thus the equation (54) and equation (55) are given by:

$$
\|\boldsymbol{N}\|=1 \Leftrightarrow 3 \gamma_{1}^{2}+2 \gamma_{1} \gamma_{2}+\gamma_{2}^{2}=1
$$

$$
\begin{aligned}
& g(\boldsymbol{\varepsilon})=\gamma_{1} \operatorname{tr}\left(\boldsymbol{\varepsilon}^{e}\right)+\gamma_{2} \varepsilon_{11}^{e}=0 \\
& \Rightarrow \gamma_{1} \varepsilon_{V}^{e}+\gamma_{2} \varepsilon_{11}^{e}=0
\end{aligned}
$$

Immediately, two particular cases must be analyzed:

- In the first case, if $\gamma_{1}=1$ and $\gamma_{2}=0$, the isotropy given by equation (52) and equation (53) is recovered. - In the second case, if $\gamma_{1}=0$ and $\gamma_{2}=1$, the pure transverse isotropy is assumed and the normal to the hyperplane coincides with the normal to the transverse isotropy plane of the material. Therefore:

$$
g(\varepsilon)=\varepsilon_{11}^{e}=0
$$

That would be the simplest possible hyperplane.

Going back to the case where the bimodular and transverse isotropy responses are induced by the damage, it is still valid: $0 \leq \gamma_{1} \leq 1$ and $0 \leq \gamma_{2} \leq 1$. However, it can be supposed that the coefficients $\gamma_{1}$ and $\gamma_{2}$ are functions of the damage variables.

Thus, accordingly with equation (51) also, regarding general cases of loading, the following relationship is proposed for the hyperplane:

$$
\begin{gathered}
g\left(\boldsymbol{\varepsilon}, \boldsymbol{D}_{\boldsymbol{T}}, \boldsymbol{D}_{\boldsymbol{C}}\right)=\boldsymbol{N}\left(\boldsymbol{D}_{\boldsymbol{T}}, \boldsymbol{D}_{\boldsymbol{C}}\right) . \boldsymbol{\varepsilon}^{\boldsymbol{e}}= \\
\gamma_{1}\left(D_{1}, D_{2}\right) \varepsilon_{V}^{e}+\gamma_{2}\left(D_{1}, D_{2}\right) \varepsilon_{11}^{e}
\end{gathered}
$$

where $\gamma_{1}\left(D_{1}, D_{2}\right)=\left\{1+H\left(D_{2}\right)\left[H\left(D_{1}\right)-1\right]\right\}$ $\eta\left(D_{1}\right)+\left\{1+H\left(D_{1}\right)\left[H\left(D_{2}\right)-1\right]\right\} \eta\left(D_{2}\right)$ and $\gamma_{2}\left(D_{1}, D_{2}\right)$ $=D_{1}+D_{2}$.

The Heaveside functions employed above are given by:

$$
\begin{aligned}
& H\left(D_{i}\right)=1 \text { for } D_{i}>0 ; H\left(D_{i}\right)=0 \\
& \text { for } D_{i}=0 \quad(i=1,2)
\end{aligned}
$$

The $\eta\left(D_{1}\right)$ and $\eta\left(D_{2}\right)$ functions are defined, respectively, for the tension and compression cases, assuming for the first one that there was no previous damage of compression affecting the present traction damage variable $D_{l}$ and analogously, for the second one that has not had previous damage of tension affecting variable $D_{2}$. 
For instance, referring to tension states without previous damage of compression, the equation (59) is reduced to the following equation:

$$
\eta\left(D_{1}\right) \varepsilon_{V}^{e}+D_{l} \varepsilon_{11}^{e}=0
$$

Equation (61) can be used to identify the passage to compression states. The $\gamma_{l}=\eta\left(D_{1}\right)$ function can be obtained from equation (57):

$$
\eta\left(D_{l}\right)=\frac{-D_{1}+\sqrt{3-2 D_{1}^{2}}}{3}
$$

Analogously, referring to compression states without previous damage in tension regimes, the equation (59) is given by:

$$
\eta\left(D_{2}\right) \varepsilon_{V}^{e}+D_{2} \varepsilon_{11}^{e}=0
$$

Therefore, imposing the eq. (56) results:

$$
\eta\left(D_{2}\right)=\frac{-D_{2}+\sqrt{3-2 D_{2}^{2}}}{3}
$$

Note that, if the damage process in the material is not activated $\left(D_{1}=D_{2}=0\right)$ the eq. (59) recovers equation (53), thus the formulation satisfies the proposed condition of initially isotropic material. However, if the material is totally damaged, $D_{l}=D_{2}=1\left(\eta\left(D_{l}\right)=\eta\right.$ $\left.\left(D_{2}\right)=0\right)$ and $\gamma_{2}=2$, the equation (58) is recovered.

To visualize the hyperplane localization in the strain space, let us consider a virgin material. In this case, the hyperplane is given by equation (53) whose normal is given by the identity second order tensor $\boldsymbol{I}$, and it has the same slope related to 1,2 and 3 directions. On the other hand, if the material is completely damaged, the hyperplane $g(\varepsilon)$ (Figure 1) is coincident to the transverse isotropy local plane of the material and, therefore, the normal vector to the hyperplane is given by the transverse isotropy tensor $\boldsymbol{A}$ (Figure 2).

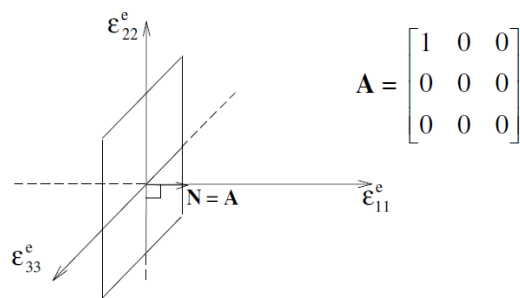

Figure 2. Hyerplane.
When the material is submitted to dominant tension state, but a previous damage process has been activated in compression, the hyperplane Equation (59) results:

$$
\left(\eta\left(D_{1}\right)+\eta\left(D_{2}\right)\right) \varepsilon_{V}^{e}+\left(D_{l}+D_{2}\right) \varepsilon_{11}^{e}=0
$$

where $\eta\left(D_{2}\right)$ and $D_{2}$ are the constants that keep the previous damage activated in compression. The same relationship is valid for dominant compression state with previous damage process activated in tension; in that case, $\eta\left(D_{1}\right)$ and $D_{1}$ are the constants that keep the previous activated damage in tension.

\section{An isotropic damage model accounting for induced bimodular elastic response}

This model has been proposed by [3] for quasi-brittle materials. It is assumed that all macroscopic effects of inelastic phenomena can be modelled by two scalar damage variables $\left(D_{T}\right.$ and $\left.D_{C}\right)$, which measure damage under stress states of prevailing tension and prevailing compression. Therefore, this model also necessitates of a criterion for partition of strain space. Besides, the model respects the principle of strain equivalence, [27].

Following the formulation, the invariant form of the constitutive model is given by:

$$
\begin{aligned}
& W(\varepsilon)=\rho \psi(\varepsilon):=\left\{\begin{array}{l}
W_{-}(\varepsilon) \text { if } \\
W_{+}(\varepsilon) \text { if }
\end{array}\right. \\
& g\left(\varepsilon, D_{T}, D_{C}\right)<0, \\
& g\left(\varepsilon, D_{T}, D_{C}\right)>0,
\end{aligned}
$$

and $W_{-}=W_{+}=W_{0}$ if $D_{T}=D_{C}=\mathbf{0}$

$$
\begin{gathered}
\mathrm{W}_{+}=\rho \psi_{+}(\varepsilon)=\frac{\lambda_{11}^{+}\left(D_{T}, D_{C}\right)}{2} \\
\operatorname{tr}^{2}(\varepsilon)+\mu_{1}\left(D_{T}, D_{C}\right) \operatorname{tr}\left(\varepsilon^{2}\right) \\
\mathrm{W}_{-}=\rho \psi_{-}(\varepsilon)=\frac{\lambda_{11}^{-}\left(D_{T}, D_{C}\right)}{2} \operatorname{tr}^{2}(\varepsilon) \\
+\mu_{1}\left(D_{T}, D_{C}\right) \operatorname{tr}\left(\varepsilon^{2}\right)
\end{gathered}
$$


where $W_{0}$ is the elastic potential of the undamaged material. The parameters $\lambda_{\mathbb{I}}^{+}, \lambda_{\mathbb{I}}^{-}$and $\mu_{1}$ are given by:

$$
\begin{gathered}
\lambda_{11}^{+}\left(D_{T}, D_{C}\right)=\lambda_{0}\left(1-D_{T}\right)\left(1-D_{C}\right) \\
\lambda_{11}^{-}\left(D_{T}, D_{C}\right)= \\
\lambda_{0}\left(1-D_{C}\right)+\frac{2}{3} \mu_{0}\left(1-D_{C}\right) D_{T} \\
\mu_{1}\left(D_{T}, D_{C}\right)=\mu_{0}\left(1-D_{T}\right)\left(1-D_{C}\right)
\end{gathered}
$$

The parameters $\lambda_{0}$ and $\mu_{0}$ are the Lamè constants for an undamaged isotropic material. In case of non-zero damage, the shear coefficients $\mu_{l}$ are the same in tension and compression as explained in [1]. However, the parameters $\lambda_{\mathbb{I}}^{+}, \lambda_{\mathbb{I}}^{-}$and $\mu_{1}$ assume different forms evidencing the bimodularity induced by damage.

The stress tensor is obtained from the gradient of the elastic potential, as follows:

$$
\begin{aligned}
& \sigma(\varepsilon)=\nabla_{\varepsilon} \rho \psi(\varepsilon)=\left\{\begin{array}{l}
\sigma_{-}(\varepsilon)=\nabla_{\varepsilon} \rho \psi_{-}(\varepsilon) \text { if } \\
\sigma_{+}(\varepsilon)=\nabla_{\varepsilon} \rho \psi_{+}(\varepsilon) \text { if }
\end{array}\right. \\
& g\left(\varepsilon, D_{T}, D_{C}\right)<0, \\
& g\left(\varepsilon, D_{T}, D_{C}\right)>0,
\end{aligned}
$$

The constitutive tensor is also obtained from the elastic potential, i.e.:

$$
\begin{aligned}
& \boldsymbol{E}(\varepsilon):=\left\{\begin{array}{l}
E_{-}(\varepsilon)=\nabla_{\varepsilon}^{2} \rho \psi_{-}(\varepsilon) \text { if } \\
E_{+}(\varepsilon)=\nabla_{\varepsilon}^{2} \rho \psi_{+}(\varepsilon) \text { if }
\end{array}\right. \\
& g\left(\varepsilon, D_{T}, D_{C}\right)<0, \\
& g\left(\varepsilon, D_{T}, D_{C}\right)>0,
\end{aligned}
$$

$$
\begin{aligned}
& E_{+}(\varepsilon)=\rho \frac{\partial^{2} \psi_{+}}{\partial \varepsilon^{2}}=E_{T}= \\
& \lambda_{11}^{+}\left(D_{T}, D_{C}\right)[I \otimes I] \\
& +2 \mu_{1}\left(D_{T}, D_{C}\right)[I \underline{\otimes} I] \\
& E_{-}(\varepsilon)=\rho \frac{\partial^{2} \psi_{-}}{\partial \varepsilon^{2}}=E_{C}= \\
& \quad \lambda_{11}^{-}\left(D_{T}, D_{C}\right)[I \otimes I] \\
& +2 \mu_{1}\left(D_{T}, D_{C}\right)[I \underline{\otimes} I]
\end{aligned}
$$

In this model, two scalar damage variables are used; therefore, a criterion is necessary to define the tension and compression of dominant states. In [3], a hyperplane $\mathrm{g}(\varepsilon)$ defined by the unit normal $N(\|N\|=1)$ has also been also used.

Following the formulation presented in this work, as mentioned before the criterion for partition of the strain space is given by eq. (51). For the isotropy case, only the tensor $\boldsymbol{A}_{\boldsymbol{I}}=\boldsymbol{I}$ is used. Therefore, the null volumetric elastic strain defines the interface between the two strain sub-domains, eqs. (52) and (53). This criterion is not dependent on the scalar damage variables $D_{T}$ and $D_{C}$ because the parameter $\gamma_{1}$ does not influence the criterion. Therefore, the criterion is expressed by eq. (53).

\section{CONCLUSIONS}

In this work, the formulation of constitutive models proposed by [1] for anisotropic elastic materials that present different behaviors in tension and compression regimes has been extended to incorporate damage processes. Although the transverse isotropy induced by damage has been studied more specifically, the formulation developed can be used for more complex cases where the material presents damage processes leading to anisotropic behavior in isotropic or anisotropic media.

As general examples, two generic damage models have been written following the formalism presented in this work. Initially, a damage model accounting for induced anisotropy and a bimodular elastic response has been described following the framework presented here. It has shown that the formulation 
of the damage model can reproduce the bimodular behavior of the material. Also, a criterion has been proposed to characterize the division of strain space. Soon after, an isotropic damage model has been presented. This model also follows the formalism of this work and it is able to reproduce the bimodular behavior of the quasi-brittle materials.

On the other hand, in Results section, two damage models found in the literature have been written following the formalism proposed in this work. These models have already been applied in the numerical simulation of concrete structures dealing with different problems ([2] and [16], for instance). To validate the proposed modelling, a plain concrete beam has been analyzed. The good performance of the models shows that the proposed formalism is able to supply consistent damage models.

Finally, the proposed framework for the damaged media can be used in future works for the development of many constitutive models depending on the involved phenomena.

\section{ACKNOWLEDGEMENTS}

The author wishes to thank to CNPq (National Council for Scientific and Technological Development) for the financial support.

\section{REFERENCES}

[1] A. Curnier, Q. He and P. Zysset. "Conewise linear elastic materials". Journal of Elasticity. Vol. 37, pp.1-38. 1995.

[2] J. J. C. Pituba and G. R. Fernandes. "An anisotropic damage model for concrete". J Eng Mech-ASCE. 137(9), 610-624, 2011. DOI: 10.1061/(ASCE)EM.1943-7889.0000260.

[3] C. Comi. "A non-local model with tension and compression damage mechanisms". Eur J Mech A-Solid. Vol. 20, pp.1-22. 2001.

[4] F. A. Araújo and S. P. B. Proença. "Application of a lumped dissipation model to reinforced concrete structures with the consideration of residual strains and cycles of hysteresis". J Mech Mater Struct. . Vol. 3, pp. 1011-1031. 2008.

[5] J. Bielski, J. J. Skrzypek and H. Kuna-Cisal. "Implementation of a model of coupled elastic-plastic unilateral damage material to finite element code”. Int J Damage Mech. Vol. 15, pp. 5-39. 2006.

[6] V. Deudée, L. Dormieux, D. Kondo and S. Maghous. "Micromechanical approach ro Nonlinear poroelasticity: application to cracked rocks". Journal of Engineering. Vol. 128, pp. 848-855. 2002

[7] Y. Liu, S. Teng and C. Soh. "Three-Dimensional Damage Model for Concrete. II: Verification", J Eng Mech-ASCE. Vol. 132, pp. 82-89. 2008.

[8] J. J. C. Pituba. "A damage model formulation: unilateral effect and RC structures analysis". Comput Concrete. 15 (5), 709-733. DOI: 10.12989/cac.2015.15.5.709, 2015.

[9] B. M. Luccioni and V. C. Rougier. "A plastic damage approach for confined concrete". Comput and Struct. Vol. 83, pp. 2238-2256. 2005.

[10] V. Pensée, D. Kondo and L. Dormieux. "Micromechanical Analysis of Anisotropic Damage in Brittle Materials". Journal of Engineering Mechanics. Vol. 128, pp. 889897. 2002.

[11] H. Welemane and F. Cormery. "Some remarks on the damage unilateral effect modeling for microcracked materials". Int J Damage Mech. Vol. 11, pp. 65-86. 2002.

[12] M. Matallah and C. La Borderie. "Inelasticitydamage-based model for numerical modeling of concrete cracking". Eng Fract Mech. Vol. 76, pp. 1087-1108. 2009.

[13] J. Mazars, Y. Berthaud and S. Ramtani. "The unilateral behaviour of damaged concrete". Engineering Fracture Mechanics. Vol. 35, pp. 629-635. 1990.

[14] D. Brancherie and A. Ibrahimbegovic. "Novel anisotrtopic continuum-discrete damage model capable of representing localized failure of massive structures. Part I: theoretical formulation and numerical implementation". International Journal of Engineering Computations. Vol. 26, pp. 100-127. 2009.

[15] A.A. Pisano, P. Fuschi and D. De Domenico. "A kinematic approach for peak load evaluation of concrete elements". Comput and Struct. Vol. 119, pp. 125-139. 2013.

[16] J. J. C. Pituba. "Anisotropic damage model on the effects of damage process due to shearing stress in concrete". Acta Sci-Technol. Vol. 
35 Issue 2, pp. 227-236. DOI: 10.4025/ actascitechnol.v35i2.13332. 2013.

[17] J.J.C. Pituba and E. A. Souza Neto. "Modeling of unilateral effect in brittle materials by a mesoscopic scale approach". Comput Concrete. Vol. 15 Issue 5, pp. 735-758. DOI: 10.12989/cac.2015.15.5.735, 2015.

[18] G. Z. Voyiadjis, Z. N. Taqieddin and P. I. Kattan. "Anisotropic damage-plasticity model for concrete". Int J. Plast. Vol. 24, pp. 1946-65. 2008.

[19] J.J.C. Pituba, G.R. Fernandes and E.A. Souza Neto. "Modelling of cohesive fracture and plasticity processes in composite microstructures". J Eng. Mech-ASCE. 142, 04016069-1 - 04016069-15. 2016.

[20] Q. Zhu, D. Kondo, J. Shao and V. Pensee. "Micromechanical modelling of anisotropic damage in brittle rocks and application". Int. J. Rock Mech Min. Vol. 45, pp. 467-477. 2008.

[21] G.R. Fernandes, J.J. C. Pituba and E.A. Souza Neto. "Multi-scale modelling for bending analysis of heteregeneous plates by coupling BEM and FEM". Eng Anal with Boundary Elem. Vol. 51 Issue 2, pp. 1-13, DOI: 10.1016/j. enganabound.2014.10.005. 2015.

[22] G. R. Fernandes, J. J. C. Pituba and E. A. Souza Neto. "FEM/BEM formulation for multi-scale analysis of stretched plates". Eng Anal with Boundary Elem. Vol. 54 Issue 2, pp. 47-59, DOI: 10.1016/j.enganabound.2014.01.005. 2015.

[23] E. Gal and R. Kryvoruk. "Fiber reinforced concrete properties - a multiscale approach". Comput Concrete. Vol. 8 Issue 5, pp. 525539. 2011.

[24] S. M. Giusti, P. J. Blanco, E. A. Souza Neto and R. A. Feijóo. "An assessment of the Gurson yield criterion by a computational multi-scale approach". Eng Computation. Vol. 26, pp. 281-301. 2009.

[25] W.F. Santos, G.R. Fernandes and J.J.C. Pituba. "Analysis of the influence of plasticity and fracture processes on the mechanical behavior of metal matrix composites microstructures". Revista Matéria. Vol. 21 Issue 3, pp. 577-598. 2016.

[26] V.P. Nguyen, O. Lloberas Valls, M. Stroeven and L.J. Sluys. "On the existence of representative volumes for softening quasibrittle materials - a failure zone averaging scheme". Comput. Methods Appl. Mech. Engrg. Vol. 199 Issue 45-48, pp. 3026-3036. 2010.

[27] J.J.C. Pituba. "On the formulation of a damage model for the concrete (in portuguese)". Sao Carlos School of Engineering. University of Sao Paulo. 2003.

[28] B. Pichler and L. Dormieux. "Instability during cohesive zone growth". Eng Fract Mech. Vol. 76, pp. 1729-1749. 2009.

[29] J. Lemaitre. "A course on damage mechanics". Springer Verlag. 1996.

[30] A.J.M. Spencer. "Continuum mechanics". Longman Scientific \& Technical. 1980.

[31] G. P. Del Piero. "Some properties of the set of fourth-order tensors, with application to elasticity". Journal of Elasticity. Vol. 9, pp. 245-261. 1979.

\section{APPENDIX}

There are three fourth-order isotropic tensors linearly independents, [30]:

$$
\begin{gathered}
\boldsymbol{I} \otimes \boldsymbol{I}=\delta_{i j} \delta_{k l}\left(e_{i} \otimes e_{j} \otimes e_{k} \otimes e_{\nu}\right) \\
\boldsymbol{I} \otimes \boldsymbol{I}=\delta_{i k} \delta_{j l}\left(e_{i} \otimes e_{j} \otimes e_{k} \otimes e_{\nu}\right) \\
\boldsymbol{I} \otimes \boldsymbol{I}=\delta_{i l} \delta_{j k}\left(e_{i} \otimes e_{j} \otimes e_{k} \otimes e_{\nu}\right)
\end{gathered}
$$

A tensor derived from the previous ones is the following:

$$
\underset{-}{-} \underset{-}{\otimes}=\frac{1}{2}[\underset{-}{\mathbf{I} \otimes \mathbf{I}}+\mathbf{I} \otimes \mathbf{I}]
$$

In what follows, the characteristic properties of those fourth-order isotropic tensors are presented. In this context, let us consider a second-order tensor $S$, which is multiplied by the fourth-order isotropic tensors:

$$
(\boldsymbol{I} \otimes \boldsymbol{I}) \boldsymbol{S}=(\operatorname{tr} \boldsymbol{S}) \boldsymbol{I}=(\boldsymbol{S} . \boldsymbol{I}) \boldsymbol{I}
$$




$$
\begin{aligned}
& (I \otimes I) S=S \\
& (I \otimes I) S=S^{T} \\
& (I \bar{\otimes} \boldsymbol{I}) S==\frac{1}{2}\left(S+S^{T}\right)
\end{aligned}
$$

Taking into account the tensors properties, the followings fourth-order tensors involved in the formulation can be derived:

$$
\begin{gathered}
\boldsymbol{A} \otimes \boldsymbol{A}=A_{i j} A_{k l}\left(e_{i} \otimes e_{j} \otimes e_{k} \otimes e_{\nu}\right) \\
\boldsymbol{A} \otimes \boldsymbol{I}=A_{i j} \delta_{k l}\left(e_{i} \otimes e_{j} \otimes e_{k} \otimes e_{\nu}\right) \\
\boldsymbol{I} \otimes \boldsymbol{A}=\delta_{i j} A_{k l}\left(e_{i} \otimes e_{j} \otimes e_{k} \otimes e_{\nu}\right) \\
\stackrel{-}{\otimes} \underset{-}{-}=\frac{1}{2}\left(A_{i k} \delta_{j l}+\delta_{i l} A_{j k}\right) \\
\left(e_{i} \otimes e_{j} \otimes e_{k} \otimes e_{\nu}\right)
\end{gathered}
$$

$$
\begin{aligned}
& \underset{-}{\boldsymbol{I}} \underset{-}{\boldsymbol{A}}=\frac{1}{2}\left(\delta_{i k} A_{j l}+A_{i l} \delta_{j k}\right) \\
& \left(e_{i} \otimes e_{j} \otimes e_{k} \otimes e_{\nu}\right)
\end{aligned}
$$

It can also be summarized the operations for dyadic products application including any second-order tensor, which were described in [1] and [31]:

$$
[R \otimes T] X=(X . T) R \forall X \in \xi
$$

$$
\begin{aligned}
& {[R \otimes T] X=R X T^{T} ;[R \otimes T] X=R^{T} T^{T}} \\
& {[R \bar{\otimes} \otimes] X=\left(R X T^{T}+X^{T} R^{T}\right) / 2} \\
& R \underline{\boldsymbol{\otimes}} \underline{T}=[R \underline{\otimes} \boldsymbol{T}+\boldsymbol{T} \bar{\otimes} \boldsymbol{R}] / 2 \\
& \forall \boldsymbol{X} \in \xi
\end{aligned}
$$

where $\xi$ is a second order symmetric tensors space. 